\title{
Semi-invariant warped product submanifolds of almost contact manifolds
}

\author{
Falleh R Al-Solamy ${ }^{1}$ and Meraj Ali Khan ${ }^{2^{*}}$
}

\author{
* Correspondence: meraj79@gmail. \\ com \\ 2Department of Mathematics, \\ University of Tabuk, Tabuk, \\ Kingdom of Saudi Arabia \\ Full list of author information is \\ available at the end of the article
}

\begin{abstract}
In this article, we have obtained necessary and sufficient conditions in terms of canonical structure $F$ on a semi-invariant submanifold of an almost contact manifold under which the submanifold reduced to semi-invariant warped product submanifold. Moreover, we have proved an inequality for squared norm of second fundamental form and finally, an estimate for the second fundamental form of a semi-invariant warped product submanifold in a generalized Sasakian space form is obtained, which extend the results of Chen, Al-Luhaibi et al., and Hesigawa and Mihai in a more general setting.
\end{abstract}

2000 Mathematics Subject Classification: 53C25; 53C40; 53C42; 53D15.

Keywords: warped product, semi-invariant, almost contact metric manifold, generalized Sasakian space-form

\section{Introduction}

Bishop and O'Neil [1] introduced the notion of warped product manifolds. These manifolds are generalization of Riemannian product manifolds and occur naturally, e.g., surface of revolution is a warped product manifold. With regard to physical applications of these manifolds, one may realize that space time around a massive star or a black hole can be modeled on a warped product manifolds for instance and warped product manifolds are widely used in differential geometry, Physics and as well as in different branches of Engineering. Due to wide applications of warped product submanifolds, this becomes a fascinating and interesting topic for research, and many articles are available in literature (c.f., [2-4]). CR-warped product was introduced by Chen [5]. He studied warped products CR-submanifolds in the setting of Kaehler manifolds and showed that there does not exist warped product CR-submanifolds of the form $M_{\perp} \times_{f} M_{T}$, therefore, he considered warped product CR-submanifolds of the types $M_{T} \times_{f} M_{\perp}$ and established a relationship between the warping function $f$ and the squared norm of the second fundamental form of the CR-warped product submanifolds in Kaehler manifolds [5]. In the available literature, many geometers have studied warped products in the setting of almost contact metric manifolds (c.f., [6-8]). Hesigawa and Mihai [9] obtained the inequality for squared norm of the second fundamental form in term of the warping function for contact CR-warped product in Sasakian manifolds. Recently Atceken [4] studied contact CR-warped product submanifolds in Cosymplectic space-forms and obtained an inequality for second fundamental form in terms of warping function. After reviewing the literature, we realized that there is very few studies on the warped product

(C) 2012 Al-Solamy and Khan; licensee Springer. This is an Open Access article distributed under the terms of the Creative Commons Attribution License (http://creativecommons.org/licenses/by/2.0), which permits unrestricted use, distribution, and reproduction in any medium, provided the original work is properly cited. 
submanifold for almsot contact manifolds so it will be worthwhile to study the warped product submnifolds in the setting of almost contact metric manifold. Since generalized Sasakian space forms include all the classes of almost contact metric manifold, so we have obtained an inequality for squared norm of second fundamental form for semiinvariant warped product submanifolds in the setting of generalized Sasakian space form.

\section{Preliminaries}

Let $\bar{M}$ be a $(2 n+1)$-dimensional $C^{\infty}$-differentiable manifold endowed with the almost contact metric structure $(\varphi, \xi, \eta, g)$, where $\varphi$ is a tensor field of type $(1,1), \xi$ is a vector field, $\eta$ is a 1 -form and $g$ is a Riemannian metric on $\bar{M}$, all these tensor fields satisfying.

$$
\begin{aligned}
& \phi^{2} X=-X+\eta(X) \xi, \quad \eta(\xi)=1, \quad g(X, \xi)=\eta(X) \\
& g(\phi X, \phi Y)=g(X, Y)-\eta(X) \eta(Y) \\
& \phi \xi=0, \quad \eta \phi=0, \quad g(X, \phi Y)=-g(\phi X, Y),
\end{aligned}
$$

for any $X, Y \in T \bar{M}$. Here, $T \bar{M}$ is the standard notation for the tangent bundle of $\bar{M}$. The two-form $\Phi$ denotes the fundamental two-form and is given by $g(X, \varphi Y)=\Phi(X, Y)$. The manifold $\bar{M}$ is said to be contact if $\Phi=d \eta$.

Most of the geometric properties of a Riemannian manifold depend on the curvature tensor $R$ of a manifold. It is well known that the sectional curvatures of a manifold determine curvature tensor completely. A Riemannian manifold with constant sectional curvature $c$ is known as real space form and its curvature tensor is given by

$$
R(X, Y) Z=c\{g(Y, Z) X-g(X, Z) Y\} .
$$

A Sasakian manifold with constant $\varphi$-sectional curvatures is a Sasakian space form and it has a specific form of its curvature tensor. Similar notion also holds for Kenmotsu and Cosymplectic space form. In order to generalize the notion in a common frame, Alegere et al. [10] introduced the notion of generalized Sasakian space form. In this connection, a generalized Sasakian space form is defined as follows.

Given an almost contact metric manifold $\bar{M}(\phi, \xi, \eta, g)$, we say that $\bar{M}$ is a generalized Sasakian space form if there exist three functions $f_{1}, f_{2}$, and $f_{3}$ on $\bar{M}$ such that, the curvature tensor $R$ is given by

$$
\begin{aligned}
& R(X, Y) Z=f_{1}\{g(Y, Z) X-g(X, Z) Y\} \\
& +f_{2}\{g(X, \phi Z) \phi Y-g(Y, \phi Z) \phi X+2 g(X, \phi Y) \phi Z\} \\
& \quad+f_{3}\{\eta(X) \eta(Z) Y-\eta(Y) \eta(Z) X \\
& \quad+g(X, Z) \eta(Y) \xi-g(Y, Z) \eta(X) \xi\} .
\end{aligned}
$$

Let $M$ be a submanifold of an almost contact metric manifold $\bar{M}$. Then we denote the induced metric on $M$ by the same symbol $g$ where as the induced connection on $M$ by $\nabla$. With these notation, Gauss and Weingarten formulae are written as

$$
\bar{\nabla}_{X} Y=\nabla_{X} Y+h(X, Y)
$$




$$
\bar{\nabla}_{X} N=-A_{N} X+\nabla_{X}^{\perp} N
$$

for each $X, Y \in T M$ and $N \in T^{\perp} M$, where $\nabla^{\perp}$ denotes the induced connection on the normal bundle $T^{\perp} M . h$ and $A_{N}$ are the second fundamental form and the shape operator of the immersion of $M$ into $\bar{M}$ and they are related as

$$
g(h(X, Y), N)=g\left(A_{N} X, Y\right) .
$$

For any $X \in T M$ and $N \in T^{\perp} M$, we write

$$
\begin{aligned}
& \phi X=T X+F X \\
& \phi N=t N+f N,
\end{aligned}
$$

where $T X$ and $t N$ are the tangential components of $\varphi X$ and $\varphi N$, respectively, where as $F X$ and $f N$ are the normal components of $\varphi X$ and $\varphi N$, respectively.

The covariant derivative of the tensors $T, F, t$, and $f$ are defined as

$$
\begin{aligned}
& \left(\bar{\nabla}_{X} T\right) Y=\nabla_{X} T Y-T \nabla_{X} Y \\
& \left(\bar{\nabla}_{X} F\right) Y=\nabla_{X}^{\perp} F Y-F \nabla_{X} Y \\
& \left(\bar{\nabla}_{X} t\right) N=\nabla_{X} t N-t \nabla_{X}^{\perp} N \\
& \left(\bar{\nabla}_{X} f\right) N=\nabla_{X}^{\perp} f N-f \nabla_{X}^{\perp} N .
\end{aligned}
$$

On the other hand, the covariant derivative of the second fundamental form $h$ is defined as

$$
\left(\bar{\nabla}_{X} h\right)(Y, Z)=\nabla^{\perp} h(Y, Z)-h\left(\nabla_{X} Y, Z\right)-h\left(Y, \nabla_{X} Z\right),
$$

for any $X, Y, Z \in T M$. Let $\bar{R}$ and $R$ be the curvature tensors of the connections $\bar{\nabla}$ and $\nabla$ on $\bar{M}$ and $M$, respectively. Then the equations of Gauss and Coddazi are given by

$$
\begin{aligned}
\bar{R}(X, Y, Z, W)= & R(X, Y, Z, W)-g(h(X, Z), h(Y, W)) \\
& +g(h(X, W), h(Y, Z)) \\
{[\bar{R}(X, Y) Z]^{\perp}=} & \left(\bar{\nabla}_{X} h\right)(Y, Z)-\left(\bar{\nabla}_{Y} h\right)(X, Z) .
\end{aligned}
$$

A submanifold $M$ of $\bar{M}$ is said to be semi-invariant submanifold if there exist on $M$, a differentiable invariant distribution $D$ such that its orthogonal complementary distribution $D^{\perp}$ is anti-invariant, i.e., $\varphi D_{x} \in T_{x} M$ and $\phi D_{x}^{\perp} \subset T_{x}^{\perp} M$ for each $x \in M$. For a semi-invariant submanifold of an almost contact metric manifold $\bar{M}$, we have

$$
T M=D \oplus D^{\perp} \oplus\langle\xi\rangle .
$$

The structure vector field $\xi$ is tangential to submanifold $M$, otherwise the submanifold is simply anti-invariant. 


$$
T^{\perp} M=\phi D^{\perp} \oplus \mu,
$$

where $\mu$ denotes the orthogonal complemantry distribution of $\varphi D^{\perp}$ and is an invariant normal subbundle of $T^{\perp} M$ under $\varphi$.

The orthogonal projection on $T M$ of a semi-invariant submanifold $M$ of an almost contact metric manifold are denoted by $P_{1}$ and $P_{2}$, i.e., for any $X \in T M$ we have

$$
X=P_{1} X+P_{2} X+\eta(X) \xi,
$$

where $P_{1} X \in D, P_{2} X \in D^{\perp}$ and $\eta(X) \xi \in\langle\xi\rangle$. It follows immediately that

$$
(a) T P_{2}=0 ; \quad(b) F P_{1}=0 ; \quad(c) t\left(T^{\perp} M\right)=D^{\perp} ; \quad(d) f T^{\perp} M \subseteq \mu .
$$

Moreover, for any $X, Y \in T M$ if we denote by $\mathcal{P}_{X} Y$ and $\mathcal{Q}_{X} Y$, the tangential and normal parts of $\left(\bar{\nabla}_{X} \phi\right) Y$, then we have

$$
\left(\bar{\nabla}_{X} \phi\right) Y=\mathcal{P}_{X} Y+\mathcal{Q}_{X} Y
$$

and on using Equations (2.5)-(2.11), we obtain

$$
\begin{aligned}
& \mathcal{P}_{X} Y=\left(\bar{\nabla}_{X} T\right) Y-A_{F Y} X-\operatorname{th}(X, Y) \\
& \mathcal{Q}_{X} Y=\left(\bar{\nabla}_{X} F\right) Y+h(X, T Y)-f h(X, Y) .
\end{aligned}
$$

Definition 2.1 A semi-invariant submanifold $M$ of an almost contact metric manifold is semi-invariant product if the distributions are involutive and their leaves are totally geodesic in $M$.

Definition 2.2 Let $\left(B, g_{B}\right)$ and $\left(F, g_{F}\right)$ be two Riemannian manifolds with Riemannian metric $g_{B}$ and $g_{F}$, respectively, and $f$ be a positive differentiable function on $B$. The warped product of $B$ and $F$ is the Riemannian manifold $(B \times F, g)$, where

$$
g=g_{B}+f^{2} g_{F} .
$$

For a warped product manifold $N_{1} \times_{f} N_{2}$, we denote by $D_{1}$ and $D_{2}$ the distributions defined by the vectors tangent to the leaves and fibers, respectively. In other words, $D_{1}$ is obtained by the tangent vectors of $N_{1}$ via the horizontal lift and $D_{2}$ is obtained by the tangent vectors of $N_{2}$ via vertical lift. In case of semi-invariant warped product submanifolds $D_{1}$ and $D_{2}$ are replaced by $D$ and $D^{\perp}$, respectively.

The warped product manifold $(B \times F, g)$ is denoted by $B \times_{f} F$. If $X$ is the tangent vector field to $M=B \times_{f} F$ at $(p, q)$ then

$$
\|X\|^{2}=\left\|d \pi_{1} X\right\|^{2}+f^{2}(p)\left\|d \pi_{2} X\right\|^{2} .
$$

Bishop and O'Neill [1] proved the following

Theorem 2.1 Let $M=B \times_{f} F$ be warped product manifolds. If $X, Y \in T B$ and $V, W \in T F$ then

(i) $\nabla_{X} Y \in T B$

(ii) $\nabla_{X} V=\nabla_{V} X=\left(\frac{X f}{f}\right) V$,

(iii) $\nabla_{V} W=\frac{-g(V, W)}{f} \nabla f$. 
$\nabla f$ is the gradient of $f$ and is defined as

$$
g(\nabla f, X)=X f,
$$

for all $X \in T M$.

Corollary 2.1 On a warped product manifold $M=N_{1} \times_{f} N_{2}$, the following statements hold

(i) $N_{1}$ is totally geodesic in $M$

(ii) $N_{2}$ is totally umbilical in $M$.

Moreover,

$$
\nabla_{X} Z=\nabla_{Z} X=(X \ln f) Z
$$

and

$$
\operatorname{nor}\left(\nabla_{Z} W\right)=\frac{-g(Z, W)}{f} \nabla f
$$

for any $X \in D_{1}$ and $Z, W \in D_{2}$, where nor $\left(\nabla_{Z} W\right)$ denotes the component of $\nabla_{Z} W$ in $D_{1}$ and $\nabla f$ denotes the gradient of $f$.

\section{Semi-invariant warped product submanifolds}

Chen [11] obtained various conditions under which a CR-submanifolds reduces to a CR-product. In particular, he proved that a CR-submanifold of a Kaehler manifold is a CR-product if and only if $\bar{\nabla} P=0$. Since, warped products are the generalization of Riemannian products by taking this point Khan et al. [8] proved a characterization of CR-warped product of a Kaehler manifold in terms of $P$ and $F$ after that Al-Luhaibi et al. [7] find charactraziation of CR-warped of nearly Kaehler manifolds in terms of $P$ and $F$. In this section, we have obtained necessary and sufficient condition in terms of $F$, for which a semi-invariant submanifolds is a semi-invariant warped product submanifold in the setting of almost contact metric manifolds.

Throughout, this section, we denote $N_{T}$ and $N_{\perp}$ the invariant and anti-invariant submanifolds, respectively, of an almost contact metric manifold $\bar{M}$. Warped product semi-invariant and semi-invariant warped product submanifolds of an almost contact metric manifold $\bar{M}$ are represented by $N_{\perp} \times_{f} N_{T}$ and $N_{T} \times_{f} N_{\perp}$ and we take $N_{T}$ tangential to $\xi$.

In terms of canonical structure $F$, we have the following charectrization.

Theorem 3.1 A semi-invariant submanifold $M$ with involutive distributions $D \oplus\langle\xi\rangle$ and $D^{\perp}$ of an almost contact metric manifold with $\mathcal{Q}_{D} D^{\perp} \in \mu$. Then $M$ is a semiinvariant warped product submanifold of $\bar{M}$ if and only if

$$
\begin{aligned}
g\left(\left(\bar{\nabla}_{U} F\right) V, \phi W\right)=- & \left(P_{1} V \mu\right) g\left(P_{2} U, W\right)+g\left(\mathcal{Q}_{P_{2} U} P_{2} V, \phi W\right) \\
& +\eta(U) g\left(\mathcal{Q}_{\xi} P_{2} V, \phi W\right)+\eta(V) g\left(\mathcal{Q}_{P_{2} U} \xi, \phi W\right),
\end{aligned}
$$

for each $U, V \in T M$ and $\mu$ a $C^{\infty}$ function on $M$ satisfying $Z \mu=0$ for each $Z \in D^{\perp}$.

Proof. Let $M$ be a semi-invariant warped product submanifold $N_{T} \times_{f} N_{\perp}$, then, by Equation (2.11), we have 


$$
g\left(\left(\bar{\nabla}_{X} F\right) Y, \phi W\right)=-g\left(\nabla_{X} Y, W\right),
$$

for any $X, Y \in D$ and $W \in D^{\perp}$. As $N_{T}$ is totally geodesic in $M$, we get

$$
g\left(\left(\bar{\nabla}_{X} F\right) Y, \phi W\right)=0 .
$$

On other hand, for any $X \in D, Z, W \in D^{\perp}$ by Equation (2.23)

$$
g\left(\left(\bar{\nabla}_{X} F\right) Z, \phi W\right)=g\left(\mathcal{Q}_{X} Z, \phi W\right) .
$$

By the assumption that $\mathcal{Q}_{D} D^{\perp} \in \mu$, the above equation gives

$$
g\left(\left(\bar{\nabla}_{X} F\right) Z, \phi W\right)=0 .
$$

As $\xi$ is tangential to $N_{T}$, for any $X \in D$ and $Z, W \in D^{\perp}$, by Equations (2.11) and (2.25) we have

$$
g\left(\left(\bar{\nabla}_{Z} F\right) X, \phi W\right)=-X \ln f g(Z, W) .
$$

Similarly, for any $Z, W, W \in D^{\perp}$, by Equation (2.11) we have

$$
g\left(\left(\bar{\nabla}_{Z} F\right) W^{\prime}, \phi W\right)=g\left(\mathcal{Q}_{Z} W^{\prime}, \phi W\right) .
$$

Moreover, for any $X \in D, W \in D^{\perp}$ and $\xi \in\langle\xi\rangle$, by Equations (2.23) and (2.25), it is easy to see that

$$
\begin{aligned}
& g\left(\left(\bar{\nabla}_{Z} F\right) \xi, \phi W\right)=g\left(\mathcal{Q}_{Z} \xi, \phi W\right) . \\
& g\left(\left(\bar{\nabla}_{\xi} F\right) Z, \phi W\right)=g\left(\mathcal{Q}_{\xi} Z, \phi W\right) .
\end{aligned}
$$

Since $\xi$ is tangential to $N_{T}$, then from Equations (2.11) and (2.25), we can prove the following

$$
\begin{aligned}
& g\left(\left(\bar{\nabla}_{\xi} F\right) X, \phi W\right)=0 . \\
& g\left(\left(\bar{\nabla}_{X} F\right) \xi, \phi W\right)=0 . \\
& g\left(\left(\bar{\nabla}_{\xi} F\right) \xi, \phi W\right)=0 .
\end{aligned}
$$

For any $U, V \in T M$ with the help of Equation (2.19), we have

$$
\begin{aligned}
g\left(\left(\bar{\nabla}_{U} F\right) V, \phi W\right)=g(( & \left.\left.\bar{\nabla}_{P_{1} U} F\right) P_{1} V, \phi W\right)+g\left(\left(\bar{\nabla}_{P_{1} U} F\right) P_{2} V, \phi W\right)+g\left(\left(\bar{\nabla}_{P_{2} U} F\right) P_{1} V, \phi W\right) \\
& +g\left(\left(\bar{\nabla}_{P_{2} U} F\right) P_{2} V, \phi W\right)+\eta(V) g\left(\left(\bar{\nabla}_{P_{1} U} F\right) \xi, \phi W\right) \\
& +\eta(V) g\left(\left(\bar{\nabla}_{P_{2} U} F\right) \xi, \phi W\right)+\eta(U) g\left(\left(\bar{\nabla}_{\xi} F\right) P_{1} V, \phi W\right) \\
& +\eta(U) g\left(\left(\bar{\nabla}_{\xi} F\right) P_{2} V, \phi W\right)+\eta(U) \eta(V) g\left(\left(\bar{\nabla}_{\xi} F\right) \xi, \phi W\right) .
\end{aligned}
$$

In view of Equations (3.2)-(3.10), the above equation reduced to Equation (3.1).

Conversely, suppose that $M$ be a semi-invariant submanifold, satisfying Equation (3.1). Then for any $X, Y \in D \oplus\langle\xi\rangle$ by Equation (3.1), we have

$$
g\left(\left(\bar{\nabla}_{X} F\right) Y, \phi W\right)=0,
$$


therefore, by Equation (2.11) the above equation yields $g\left(\nabla_{X} Y, W\right)=0$, this mean leaves of $D \oplus\langle\xi\rangle$ are totally geodesic in $M$.

Now, for any $Z, W \in D^{\perp}$, by Equation (3.1), we get

$$
g\left(\left(\bar{\nabla}_{Z} F\right) X, \phi W\right)=-(X \mu) g(Z, W),
$$

or

$$
g\left(\nabla_{Z} W, X\right)=-(X \mu) g(Z, W) .
$$

Let $N_{\perp}$ be a leaf of $D_{\perp}$. If $\nabla^{\prime}$ denotes the induced connection on $N_{\perp}$ and $h^{\prime}$ be the second fundamental form of the immersion of $N_{\perp}$ of $M$, then by Gauss formula

$$
g\left(X, \nabla_{Z}^{\prime} W+h^{\prime}(Z, W)\right)=-(X \mu) g(Z, W)
$$

or

$$
g\left(X, h^{\prime}(Z, W)\right)=-g(X, \nabla \mu) g(Z, W)
$$

or

$$
h^{\prime}(Z, W)=-\nabla \mu g(Z, W),
$$

this shows that $N_{\perp}$ is totally umbilical in $M$ with mean curvature vector $\nabla \mu$. Moreover, as $W \mu=0$ for all $W \in D^{\perp}$ and the mean curvature is parallel on $N^{\perp}$, this shows that $N_{\perp}$ is extrinsic sphere. Hence, by virtue of result of [12] which says that if the tangent bundle of a Riemannian manifold $M$ splits into an orthogonal sum $T M=E_{0} \oplus E_{1}$ of nontrivial vector subbundles such that $E_{1}$ is spherical and its orthogonal complement $E_{0}$ is auto parallel, then the, manifold $M$ is locally isometric to a warped product $M_{0} \times_{f} M_{1}$, we can say $M$ is a locally semi-invariant product submanifold $N_{T} \times_{f} N_{\perp}$, where warping function $f=e^{\mu}$.

Let $M=N_{T} \times_{f} N_{\perp}$ be a semi-invariant warped product submanifold of an almost contact metric manifold $\bar{M}$.

In view of decomposition (2.18), we may write

$$
h(U, V)=h_{\phi D^{\perp}}(U, V)+h_{\mu}(U, V),
$$

for each $U, V \in T M$, where $h_{\phi D^{\perp}}(U, V) \in \phi D^{\perp}$ and $h_{\mu}(U, V) \in \mu$.

If $\left\{e_{1}, e_{2}, \ldots, e_{n}\right\}$ be a local orthonormal frame of vector fields on $M$ then we define

$$
\|h\|^{2}=\sum_{i, j=1}^{n} g\left(h\left(e_{i}, e_{j}\right), h\left(e_{i}, e_{j}\right)\right),
$$

and for differentiable function $f$ on $M$, the Laplacian $\Delta f$ of $f$ is defined as

$$
\Delta f=\sum_{j=1}^{n}\left\{e_{j}\left(e_{j} f\right)-\nabla_{e_{j}} e_{j} f\right\} .
$$

Now we have the following proposition

Proposition 3.1 Let $M$ be a semi-invariant warped product submanofold $N_{T} \times{ }_{f} N_{\perp}$ of an almost contact metric manifold of $\bar{M}$. Then 
(i) $h_{\phi D^{\perp}}(\phi X, Z)=(X \ln f) \phi Z+\phi \mathcal{P}_{Z} \phi X$

(ii) $g\left(\mathcal{Q}_{Z} X, \phi W\right)=g\left(\mathcal{P}_{Z} \phi X, W\right)$

(iii) $g\left(h(\phi X, Z, \phi h(X, Z))=\left\|h_{\mu}(X, Z)\right\|^{2}-g\left(\mathcal{Q}_{X} Z, \phi h_{\mu}(X, Z)\right)\right.$, for any $X \in T N_{T}$ and $Z, W \in T N_{\perp}$.

Proof. By Gauss formula

$$
h(\phi X, Z)=\left(\bar{\nabla}_{Z} \phi\right) X+\phi \nabla_{Z} X+\phi h(X, Z)-\nabla_{Z} \phi X,
$$

using the decomposition (2.21) and Equation (2.25), we get

$$
h(\phi X, Z)=\mathcal{P}_{Z} X+\mathcal{Q}_{Z} X+(X \ln f) \phi Z+\phi h(X, Z)-(\phi X \ln f) Z .
$$

Comparing tangential parts in above equation

$$
\mathcal{P}_{Z} X=(\phi X \ln f) Z-\phi h_{\phi D^{\perp}}(X, Z),
$$

taking inner product with $W \in D^{\perp}$ on both side, we get

$$
g(h(X, Z), \phi W)=-\phi X \ln f g(Z, W)+g\left(\mathcal{P}_{Z} X, W\right),
$$

or equivalently

$$
h_{\phi D^{\perp}}(X, Z)=(-\phi X \ln f) \phi Z+\phi \mathcal{P}_{Z} X,
$$

or

$$
h_{\phi D^{\perp}}(\phi X, Z)=(X \ln f) \phi Z+\phi \mathcal{P}_{Z} \phi X,
$$

which proves the part (i) of proposition.

Now, on comparing the normal parts

$$
h(\phi X, Z)=\mathcal{Q}_{Z} X+X \ln f \phi Z+\phi h_{\mu}(X, Z),
$$

or

$$
h(\phi X, Z)-\phi h_{\mu}(X, Z)=\mathcal{Q}_{Z} X+(X \ln f) \phi Z,
$$

taking inner product with $\varphi W$ and using Equation (3.17), we get

$$
g\left(\mathcal{Q}_{Z} X, \phi W\right)=g\left(\mathcal{P}_{Z} \phi X, W\right) .
$$

Taking inner product with $\varphi h(X, Z)$ in Equation (3.14), we find

$$
g(h(\phi X, Z), \phi h(X, Z))=\left\|h_{\mu}(X, Z)\right\|^{2}-g\left(\mathcal{Q}_{X} Z, \phi h_{\mu}(X, Z)\right),
$$

which is the part (iii) of proposition.

For semi-invariant warped product submaniolds of an almost conatct metric manifold, we have the following theorem

Theorem 3.2 Let $M=N_{T} \times_{f} N_{\perp}$ be a semi-invariant warped product submanifold of an almost contact manifold $\bar{M}$ with $\mathcal{P}_{D_{\perp}} D \in D$, then

(i) The squared norm of the second fundamental form satisfies

$$
\|h\|^{2} \geq 2 q\|\nabla \ln f\|^{2}+\left\|\mathcal{P}_{D_{\perp}} D\right\|^{2},
$$


where $\nabla \ln f$ is the gradient of $\ln f$ and $q$ is the dimension of anti-invariant distribution.

(ii) If the equality sign in (3.15) holds identically, then $N_{T}$ is totally geodesic submanifolds of $\bar{M}, N_{\perp}$ is a totally umbilical submanifold of $\bar{M}, M$ is minimal and $h\left(D \oplus D^{\perp}, \xi\right)=0$.

Proof. Let $\left\{X_{0}=\xi, X_{1}, X_{2}, \ldots, X_{p}, X_{p+1}=\varphi X_{1}, \ldots, X_{2 p}=\varphi X_{p}\right\}$ be a local orthonormal frame of vector field on $N_{T}$ and $\left\{Z_{1}, Z_{2}, \ldots, Z_{q}\right\}$ be a local orthonormal frame of vector field on $N_{\perp}$. Then by definition of squared norm of mean curvature vector

$$
\begin{aligned}
\|h\|^{2}=\sum_{i, j=1}^{2 p} g\left(h\left(X_{i}, X_{j}\right), h\left(X_{i}, X_{j}\right)\right)+\sum_{i=1}^{2 p} \sum_{r=1}^{q} g\left(h\left(X_{i}, Z_{r}\right), h\left(X_{i}, Z_{r}\right)\right) \\
\\
+\sum_{r, s=1}^{q} g\left(h\left(Z_{r}, Z_{s}\right), h\left(Z_{r}, Z_{s}\right)\right) \\
+\sum_{i=1}^{2 p} g\left(h\left(X_{i}, \xi\right), h\left(X_{i}, \xi\right)\right) \\
+\sum_{r=1}^{q} g\left(h\left(Z_{r}, \xi\right), h\left(Z_{r}, \xi\right)\right)
\end{aligned}
$$

Thus,

$$
\|h\|^{2} \geq \sum_{i=1}^{2 p} \sum_{r=1}^{q} g\left(h\left(X_{i}, Z_{r}\right), h\left(X_{i}, Z_{r}\right)\right) .
$$

On using part (i) of Proposition (3.1) with assumption $\mathcal{P}_{D_{\perp}} D \in D$, then the above inequality takes the form

$$
\|h\|^{2} \geq \sum_{i=1}^{2 p} \sum_{r=1}^{q}\left(\left(\phi X_{i} \ln f\right)^{2} g\left(Z_{r}, Z_{r}\right)+\left\|\mathcal{P}_{Z_{r}} X_{i}\right\|^{2} .\right.
$$

Using $\sum_{i=1}^{2 p} \sum_{r=1}^{q}\left\|\mathcal{P}_{Z_{r}} X_{i}\right\|^{2}=\left\|\mathcal{P}_{D_{\perp}} D\right\|^{2}$, the above inequality can be represented as

$$
\|h\|^{2} \geq 2 q\|\nabla \ln f\|^{2}+\left\|\mathcal{P}_{D_{\perp}} D\right\|^{2},
$$

which proves the part (i) of the Theorem.

Finally, if equality holds identically then from Equation (3.16), $h(D, D)=0, h\left(D^{\perp}, D^{\perp}\right)=0$, $h\left(D, D^{\perp}\right) \subseteq \varphi D^{\perp}$, and $h\left(D \oplus D^{\perp}, \xi\right)=0$. These observations proves the part (ii) of theorem.

\section{Semi-invariant warped product submanifolds of a generalized Sasakian space-form}

Hesigawa and Mihai [9] obtained the inequality for squared norm of second fundamental form for contact CR-warped product submanifolds in the setting of Sasakian space form. In the available literature, similar estimates are proved for squared norm of second fundamental form in contact manifolds (c.f., $[3,4]$ ). Since generalized Sasakian space form include the class of all almost contact metric manifold, so in this section we will obtain an estimate for the squared norm of second fundamental form 
for semi-invariant warped product submani-folds in the setting of generalized Sasakian space form.

Theorem 4.1 Let $M=N_{T} \times_{f} N_{\perp}$ be a semi-invariant warped product submanifold of a generalized Sasakian space form with $\mathcal{P}_{D_{\perp}} D \in D$. Then we have

$$
\|h\|^{2} \geq 2 q\left\{f_{2} p+\frac{1}{2} \Delta \ln f+\|\nabla \ln f\|^{2}\right\}
$$

Proof. For $X \in D$ and $Z \in D^{\perp}$, by formula (2.4) we have

$$
\bar{R}(X, \phi X, Z, \phi Z)=-2 f_{2} g(X, X) g(Z, Z) .
$$

On the other hand by Coddazi equation

$$
\begin{aligned}
\bar{R}(X, \phi X, Z, \phi Z)=g\left(\nabla_{X}^{\perp} h(\phi X, Z), \phi Z\right)-g\left(h\left(\nabla_{\phi X} X, Z\right), \phi Z\right) \\
-g\left(h\left(\phi X, \nabla_{X} Z\right), \phi Z\right)-g\left(\nabla_{\phi X}^{\perp} h(X, Z), \phi Z\right) \\
+g\left(h\left(\nabla_{\phi X} X, \phi Z\right)+g\left(h\left(X, \nabla_{\phi X} Z\right), \phi Z\right) .\right.
\end{aligned}
$$

Now,

$$
g\left(\nabla_{X}^{\perp} h(\phi X, Z), \phi Z\right)=X g(h(\phi X, Z), \phi Z)-g\left(h(\phi X, Z), \bar{\nabla}_{X} \phi Z\right) .
$$

The first term in the right-hand side of Equation (4.3) on using Equation (2.25), decomposition (2.25) and part (ii) of Proposition (3.1) becomes,

$$
X g(h(\phi X, Z), \phi Z)=X g(X \ln f \phi Z, \phi Z)+X g\left(\mathcal{P}_{Z} \phi X, Z\right) .
$$

In view of assumption $\mathcal{P}_{D^{\perp}} D \in D$, the above equation gives

$$
X g(h(\phi X, Z), \phi Z)=\left(X(X \ln f)+2(X \ln f)^{2}\right) g(Z, Z) .
$$

Where as, the second term of Equation (4.3) with the help of Equations (2.5) and (2.25) can be written as

$$
\begin{gathered}
g\left(h(\phi X, Z), \bar{\nabla}_{X} \phi Z\right)=g\left(h(\phi X, Z), \mathcal{Q}_{X} Z\right)+X \ln f g(h(\phi X, Z), \phi Z) \\
+g(h(\phi X, Z), \phi h(X, Z)) .
\end{gathered}
$$

By (i) and (ii) parts of Proposition 3.1, the above equation becomes

$$
g\left(h(\phi X, Z), \bar{\nabla}_{X} \phi Z\right)=g\left(h(\phi X, Z)-\phi h(X, Z), \mathcal{Q}_{X} Z\right)+(X \ln f)^{2}\|Z\|^{2}+\left\|h_{\mu}(X, Z)\right\|^{2} .
$$

Applying Equation (3.14), (i) and (ii) parts of Proposition 3.1, we get

$$
g\left(h(\phi X, Z), \bar{\nabla}_{X} \phi Z\right)=(X \ln f)^{2}\|Z\|^{2}+\left\|h_{\mu}(X, Z)\right\|^{2}-\left\|\mathcal{Q}_{X} Z\right\|^{2} .
$$

On substituting Equations (4.4) and (4.5) in Equation (4.3), we find

$$
\begin{aligned}
g\left(\nabla_{X}^{\perp} h(\phi X, Z), \phi Z\right)= & \left(X(X \ln f)+(X \ln f)^{2}\right) g(Z, Z) \\
& +\left\|\mathcal{Q}_{X} Z\right\|^{2}-\left\|h_{\mu}(X, Z)\right\|^{2} .
\end{aligned}
$$

Similarly, we obtain

$$
\begin{aligned}
g\left(\nabla_{\phi X}^{\perp} h(\phi X, Z), \phi Z\right)= & \left(\phi X(\phi X \ln f)+(\phi X \ln f)^{2}\right) g(Z, Z) \\
& +\left\|\mathcal{Q}_{\phi X} Z\right\|^{2}-\left\|h_{\mu}(X, Z)\right\|^{2} .
\end{aligned}
$$


By formula (2.25) and part (i) of Proposition 3.1, we have

$$
g\left(h\left(\phi X, \nabla_{X} Z\right), \phi Z\right)=(X \ln f)^{2} g(Z, Z)
$$

and

$$
g\left(h\left(X, \nabla_{X} Z\right), \phi Z\right)=-(\phi X \ln f)^{2} g(Z, Z) .
$$

On using (i) and (ii) parts of Proposition 3.1 and the fact $N_{T}$ is totally geodesic, we have

$$
g\left(h\left(\nabla_{\phi X} X, Z\right), \phi Z\right)=-\left(\phi \nabla_{\phi X} X(\ln f) g(Z, Z)\right.
$$

and

$$
g\left(h\left(\nabla_{X} \phi X, Z\right), \phi Z\right)=-\left(\phi \nabla_{X} \phi X(\ln f) g(Z, Z) .\right.
$$

The right-hand side of above equation, on making use of the fact that $N_{T}$ is totally geodesic in $M$ and the formula (2.25) reduced to $-g\left(\nabla_{Z} \varphi \nabla_{X} \varphi X, Z\right)$, thus by using Gauss formula, we find

$$
\begin{aligned}
g\left(h\left(\nabla_{X} \phi X, Z\right), \phi Z\right)= & \left(\left(\nabla_{X} X\right) \ln f\right) g(Z, Z)+\left(\left(\nabla_{\phi X} \phi X\right) \ln f\right) g(Z, Z) \\
& -\left(\phi \nabla_{\phi X} X \ln f\right) g(Z, Z)
\end{aligned}
$$

Let $\left\{X_{0}=\xi, X_{1}, X_{2}, \ldots, X_{p}, X_{p+1}=\varphi X_{1}, \ldots, X_{2 p}=X_{p}\right\}$ and $\left\{Z_{1}, Z_{2}, \ldots, Z_{q}\right\}$ be a local orthonormal frame of vector fields on $N_{T}$ and $N_{\perp}$, respectively. Choosing $X, Z$ as basic vector fields and substituting from Equations (4.3)-(4.10) into Equation (4.2), we obtain

$$
\begin{aligned}
\bar{R}\left(X_{i}, \phi X_{i}, Z_{r}, \phi Z_{r}\right)= & X_{i}\left(X_{i} \ln f\right) g\left(Z_{r}, Z_{r}\right)+\phi X_{i}\left(\phi X_{i} \ln f\right) g\left(Z_{r}, Z_{r}\right) \\
- & \left(\left(\nabla_{X_{i}} X_{i}\right) \ln f+\left(\nabla_{\phi X_{i}} \phi X_{i}\right) \ln f g\left(Z_{r}, Z_{r}\right)+\left\|\mathcal{Q}_{X_{i}} Z_{r}\right\|^{2}\right. \\
& +\left\|\mathcal{Q}_{\phi X_{i}} Z_{r}\right\|^{2}-\| h_{\mu}\left(X_{i}, Z_{r}\left\|^{2}-\right\| h_{\mu}\left(\phi X_{i}, Z_{r}\right) \|^{2} .\right.
\end{aligned}
$$

Summing both side over $i=1,2, \ldots, p, r=1,2, \ldots, q$ and making use of Equation (4.1), we obtain

$$
\left\|h_{\mu}\left(D, D^{\perp}\right)\right\|^{2}=\left\|\mathcal{Q}_{D} D^{\perp}\right\|^{2}+2 f_{2} p q+q \Delta \ln f .
$$

Here we use

$$
\begin{aligned}
& \left\|\mathcal{Q}_{D} D^{\perp}\right\|^{2}=\sum_{i=0}^{2 p} \sum_{r=1}^{q}\left\|\mathcal{Q}_{X_{i}} Z_{r}\right\|^{2} \\
& \left\|h_{\mu}\left(D, D^{\perp}\right)\right\|^{2}=\sum_{i=0}^{2 p} \sum_{r=1}^{q}\left\|h_{\mu}\left(X_{i}, Z_{r}\right)\right\|^{2} .
\end{aligned}
$$

Finally, on the same line of the proof of Equation (3.15), we can prove

$$
\left\|h_{\phi D^{\perp}}\left(D, D^{\perp}\right)\right\|^{2}=2 q\|\nabla \ln f\|^{2}+\left\|\mathcal{P}_{D_{\perp}} D\right\|^{2} .
$$

The result follows immideatly from Equations (4.11) and (4.12). 
Acknowledgements

Authors are thankful to the referee for his valuable suggestion and comments. This work is supported by the Research Grant number 0136-1432-S, Deanship of Scientific research, University of Tabuk, K.S.A.

\section{Author details}

'Department of Mathematics, King Abdulaziz University, P.O. Box 80015, Jeddah 21589, Kingdom of Saudi Arabia

${ }^{2}$ Department of Mathematics, University of Tabuk, Tabuk, Kingdom of Saudi Arabia

\section{Authors' contributions}

All authors read and approved the final manuscript.

\section{Competing interests}

The authors declare that they have no competing interests.

Received: 1 December 2011 Accepted: 7 June 2012 Published: 7 June 2012

\section{References}

1. Bishop, RL, O'Neill, B: Manifolds of negative curvature. Trans Am Math Soc. 145, 1-49 (1965)

2. Khan, KA, Khan, VA, Uddin, S: Warped product submanifolds of cosymplectic manifolds. Balkan J Geom Appl. 13, 55-65 (2008)

3. Arslan, K, Ezentas, R, Mihai, I, Murathan, C: Contact CR-warped product submani-folds in Kenmotsu space forms. J Korean Math Soc. 42(5), 1101-1110 (2005)

4. Atceken, M: Contact CR-warped product submanifolds in cosymplectic space forms. Collect Math. 62, 17-26 (2011). doi:10.1007/s13348-010-0002-z

5. Chen, BY: Geometry of warped product CR-submanifolds in Kaehler manifolds I. Monatsh Math. 133, 177-195 (2001). doi:10.1007/s006050170019

6. Mihai, I: Contact CR-warped product submanifolds in Sasakian space forms. Geom Dedic. 109, 165-173 (2004). doi:10.1007/s10711-004-5459-z

7. Al-Luhaibi, NS, Al-Solamy, FR, Khan, VA: CR-warped product submanifolds of nearly Kaehler manifolds. J Korean Math Soc. 46(5), 979-995 (2009)

8. Khan, VA, Khan, KA, Siraj-Uddin, : CR-warped product submanifolds in a Kaehler manifold. Southeast Asian Bull Math. 33 , 865-874 (2009)

9. Hasegawa, I, Mihai, I: Contact CR-warped product submanifolds in Sasakian manifolds. Geom Dedic. 102, 143-150 (2003)

10. Alegre, P, Blair, D, Carriazo, A: Generalized Sasakian-space-forms. Israel J Math. 14, 157-183 (2004)

11. Chen, BY: CR-submanifolds of a Kaehler manifold I. J Differ Geom. 16, 305-323 (1981)

12. Hiepko, S: Eine Inner Kenneichungder verzerrten Produkte. Math Ann. 241, 209-215 (1979). doi:10.1007/BF01421206

doi:10.1186/1029-242X-2012-127

Cite this article as: Al-Solamy and Khan: Semi-invariant warped product submanifolds of almost contact manifolds. Journal of Inequalities and Applications 2012 2012:127.

\section{Submit your manuscript to a SpringerOpen ${ }^{\odot}$ journal and benefit from:}

- Convenient online submission

Rigorous peer review

- Immediate publication on acceptance

- Open access: articles freely available online

- High visibility within the field

- Retaining the copyright to your article

Submit your next manuscript at $\gg$ springeropen.com 